\title{
EV Charging Station Placement Considering Traffic Flow Tianqi $\mathrm{Lu}^{1, \mathrm{a}}$, Qiang $\mathrm{Ma}^{2, \mathrm{~b}}$, Zheng $\mathrm{Gu}^{3, \mathrm{c}}$
}

\author{
${ }^{1}$ State Grid Liaoning Electric Power Company Limited Economic Research Institute, Shenyang, \\ China \\ ${ }^{2}$ State Grid Liaoning Electric Power Company Limited Economic Research Institute, Shenyang, \\ China \\ ${ }^{3}$ State Grid Liaoning Electric Power Company Limited Economic Research Institute, Shenyang, \\ China
}

aaemail: lutianqi5558972@sina.com, bemail:6421106@qq.com, ${ }^{c}$ email: 17186477@qq.com

Keywords: The flow refueling model; Multi-objective optimization; NSGA- II

\begin{abstract}
The flow refueling location model is adopted to describe the traffic network considering the shortest and the second shortest paths. Three objective functions of the electric vehicle charging station placement optimal model are defined to maximize the captured traffic flow, to minimize the investment cost, and to minimize the average voltage deviation. Then, the non-dominated sorting genetic algorithm- II is used to solve the multi-objective model. With the example of the IEEE 33node power distribution network and the 25-node traffic network, the basic characteristics of the presented model and solving method are illustrated.
\end{abstract}

\section{INSTRUCTIONS}

With the growing prominence of energy and environmental issues in recent years and the development of related technologies, more attention has been paid on electric vehicles (EVs) for their advantages in saving the energy and protecting the environment. EV charging stations, which are both public service facilities and power load facilities, need to be carefully planned before constructing. The influence of not only the traffic network, but also the power grid should be considered.

Many works have been done about EV charging station placement. [1] introduces planning of EV charging and charging station construction from macro point of view, including the factors affecting the layout of EV charging stations and overall principles of charging station planning.In [2],the objective is set to minimizetotal construction cost, subject to constraints of charging station coverage and convenience for drivers to charge their EVs. Literature [3] proposes a well-organized system architecture operational scheduling method for charging and discharging of EVs.Multiobjective framework aims at minimizing total operation cost and emissions.Benders decomposition technique is used to solve the proposed model.Influence of the traffic flow is included in the planning model[4], and the super-efficiency data envelopment analysis is employed to transfer multiobjective optimization into a single-objective one.

In this paper, we adopt the flow refueling location mode considering shortest and second shortest paths, to describe effect of traffic network.Three objectives of the optimal EV charging station placement model is to maximize the captured traffic flow, as well as to minimize the investment cost and average voltage deviation.

\section{EV charging station placement model}

\section{Model optimal objectives}

The optimal objectives we proposed in this paper are to maximize the traffic flow that charging stations capture, to minimize the investment cost and to minimize the power bus voltage deviation. To maximize the captured traffic flow 
Assuming every time a driver passes a charging station, he will choose to fully charge his EV.

$\operatorname{Max} f_{1}=\sum_{q \in Q} \sum_{r \in R q} f_{q} g_{q r} y_{q r}$

In (1), $q$ represents a pair of start node and terminal node; $Q$ represents all there pairs; $r$ represents the shortest path or the second shortest path; $R_{q}$ is the set of the shortest path and the second shortest path between the node pair $q ; f_{q}$ is the traffic flow on the shortest path between the node pair $q ; g_{q r}$ is the proportion of drivers who are willing to take the path between the node pair $q ; y_{q r}$ shows whether the traffic flow on path $r$ between the node pair $q$ can be captured by the charging stations on this path, $y_{q r}=1$ if the traffic flow can be captured, otherwise $y_{\text {qr }}=0$.

\section{To minimize the investment cost}

Assuming investment cost includes 3 parts: construction cost of the charging stations, cost of power capacity expansion, and cost of network power loss after charging stations are constructed.

Min $f_{2}=\sum_{k \in K} c_{1, k} X_{C S, k}+\sum_{i \in N_{i}} c_{2, i} X_{S R, i}+c_{3} P_{\text {loss }}$

Where, $k$ is the a candidate position where charging stations can be built; $K$ is the set of all the candidate positions; $x_{C S, k}$ shows whether a charging station is built at the position $k, x_{C S, k}=1$ if the charging station is built; $i$ represents a power bus; $N_{i}$ is the set of all the power buses; $x_{S R, i}$ shows whether a power bus capacity need to be expanded, $x_{S R, i}=1$ if it needs to be expanded; $P_{\text {loss }}$ is the power network loss; $c_{1, k}$ is the construction cost of building a charging station at node $k ; c_{2, i}$ is the cost of expanding the power capacity at bus $i ; c_{3}$ is the cost of network power loss; in this paper $c_{1, k}, c_{2, i}$ and $c_{3}$ are set as constants.

To minimize the voltage deviation

$$
\operatorname{Min} f_{3}=\sum_{i \in N_{i}} \gamma_{i}\left(\frac{\left|V_{i}-V_{0}\right|}{V_{0}}\right)
$$

Where, $V_{i}$ is the voltage at bus $i ; V_{0}$ is the voltage at swing bus; $\gamma_{i}$ is the factor representing the importance of bus $i$.

\section{Model constraints}

\section{Node combination constraint}

Only when the charging stations are built at a combination of nodes on a path, which meets the EVs' refueling requirements and allows them to go from the start to the end and return to the start, the traffic flow on this path can be counted as captured. In this situation, we call the combination of nodes feasible. Then we define a parameter $v_{h}$ to represent whether a combination of nodes $h$ is open. To a path $q$, if charging stations are built at every node of a feasible node combination $h$, we say the combination is open and $v_{h}=1$, otherwise $v_{h}=0$. The constraint for variable $y_{q r}$ is

$y_{q r}= \begin{cases}1 & \sum_{h \in H_{q r}} v_{h} \geq 1 \\ 0 & \sum_{h \in H_{q r}} v_{h}=\mathbf{0}\end{cases}$

Where, $H_{q r}$ is the set of all the feasible node combination on a path. Equation (4) shows that when charging stations are built at every node of at least one feasible node combination on a path, then the traffic flow on this path can be captured, and $y_{q r}=1$.

\section{Charging station number constraint}

The number of all the charging stations we plan to construct is $p$.

$$
\sum_{k \in K} x_{C S, k}=p
$$




\section{Power bus capacity constraint}

$$
x_{S R, \mathrm{i}}= \begin{cases}1 & P_{i, k}+\sum_{k \in K_{i}} x_{C S, k} P_{c, k}>P_{\mathrm{i}, \max } \\ 0 & P_{i, k}+\sum_{k \in K_{i}} x_{C S, k} P_{c, k} \leq P_{\mathrm{i}, \max }\end{cases}
$$

Where, $P_{i, k}$ is the original power load of bus $i$, which is corresponding to the traffic node $k$; $K_{i}$ is the set of traffic nodes which are corresponding to power bus $i ; P_{c, k}$ is the charging power of the charging station built at node $k ; P_{\mathrm{i}, \max }$ is the limit of active power of bus $i$.

In (6), we need to calculate the charging power of the station built at node $k$. In this paper, according to historic data, we assume the total charging power $P_{c, t o t a l}$ in the planning area is a known parameter, and the charging power of each charging station is directly proportional to the ratio between the traffic flow captured by the station and the total traffic flow.

$$
\boldsymbol{P}_{c, k}=\boldsymbol{P}_{c, \text { total }} \cdot \frac{f_{k}}{\sum_{k \in K_{s}} f_{k}}
$$

\section{Power flow equation constraint}

Power flow equation is the basic constraint in power system.

$$
\left\{\begin{array}{l}
-P_{i, k}-x_{C S, k} P_{c, k}=\sum_{j=i}^{N} V_{i, k} V_{j}\left(G_{i j} \cos \theta_{i j}+B_{i j} \sin \theta_{i j}\right) \\
-Q_{i, k}-x_{C S, k} Q_{c, k}=\sum_{j=i}^{N} V_{i, k} V_{j}\left(G_{i j} \sin \theta_{i j}-B_{i j} \cos \theta_{i j}\right)
\end{array}\right.
$$

Where, $P_{i, k}$ and $Q_{i, k}$ respectively represent the original active power and reactive power at bus $i$ which is corresponding to the traffic node $k ; P_{c, k}$ and $Q_{c, k}$ respectively represent the active and reactive power the charging station consumes at node $k ; G_{i j}$ and $B_{i j}$ are respectively the real part and imaginary part of the bus admittance matrix; $\theta_{i j}$ is the phase angle difference between the voltages of the two terminals of the line $i j$.

\section{Solving method}

Take (1)-(3) as objectives, and (4)-(8) as constraints, we get the EV charging station placement model, which is a multi-objective nonlinear integer optimization problem. To solve this model, we choose the non-dominated sorting genetic algorithm- II (NSGA- II ) ${ }^{[10]}$ as the solving method. By using this algorithm, we can get a set of pareto solutions, among which no solution can dominate the others, i.e. can be better than the others in all 3 objective directions.

\section{The flow refueling location model}

\section{The flow capturing location allocation model}

M. John Hodgson proposed the flow capturing model in $1990{ }^{[5]}$. In this model, traffic flow is moving on the road and passes the traffic nodes. Assuming there is a traffic path which starts at node $\mathrm{A}$ and ends at node $\mathrm{D}$. If at least one service facility is constructed among all the nodes from A to $\mathrm{D}$, then all the traffic flow on this path can be captured. The objective of the model is to capture the traffic as much as possible.

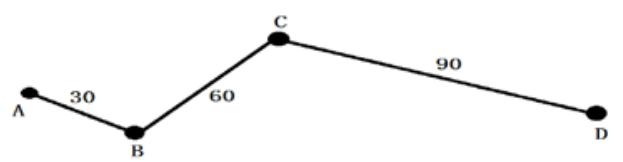

Figure 1. A path from node A to D (unit: $\mathrm{km}$ )

\section{The flow refueling model for $\mathrm{EV}$ charging stations}

The flow capturing location model in 2.1 assumes only one facility can capture all the traffic flow on the path, which avoids the repetitive counting of the captured traffic flow when more than one facility is constructed on the path.

However, when it comes to EV charging station planning, we need to consider the constraint of EVs' battery capacity, i.e. the EVs' range. For example, in figure 1, when an EV's range is between $90 \mathrm{~km}$ and $180 \mathrm{~km}$, it will require more than one charging station to refuel in order to finish a trip from $\mathrm{A}$ to $\mathrm{D}$, and back to A. In fact, the EV can finish the mentioned trip only when 3 charging stations are built at nodes (A, C, D) or (B, C, D). 
When we take the constraint of EVs' range into consideration, we need to figure out in order to capture the traffic flow on a path, at which nodes we should construct the charging stations. After knowing all the nodes a path passes and an EV's range, we can use the method in [6] to get the combination of nodes which can meet the EV's charging need to achieve a round trip.

\section{The choice of driving paths}

In the flow refueling model, an important parameter is the combination of all the nodes on the path from a start to a terminal. Considering most drivers now have navigation equipment, we assume they will choose the shortest path. Also, under some circumstances, drivers may need to take a detour to get the destination, so we assume some drivers will choose the second shortest paths.

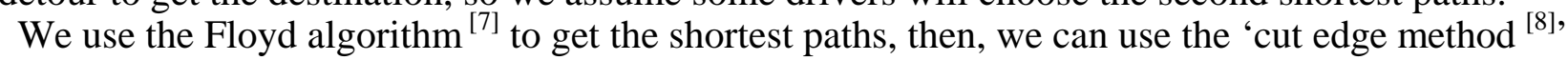
to obtain the second shortest paths.

By using the above-mentioned algorithms, we have both the shortest and second shortest paths that we need. To simulate the traffic flow on the shortest paths, we choose the simple gravity spatial interaction model ${ }^{[4]}$ to calculate. While for the second shortest paths, considering the fact that not all drivers are willing to choose them, according to the model Kim and Kuby proposed ${ }^{[9]}$, we multiply the traffic flow on the shortest paths by a path deviation factor which is smaller than 1 , to simulate the traffic flow on the second shortest paths.

\section{Simulation}

\section{Test case}

The test case used in this paper is combined by IEEE 33-bus power distribution system and 25node traffic system. And we assume the traffic nodes 1 to 25 are corresponding to the power buses 1-25. The topologies of the power distribution system and the traffic system are shown in figure 2 and figure 3.

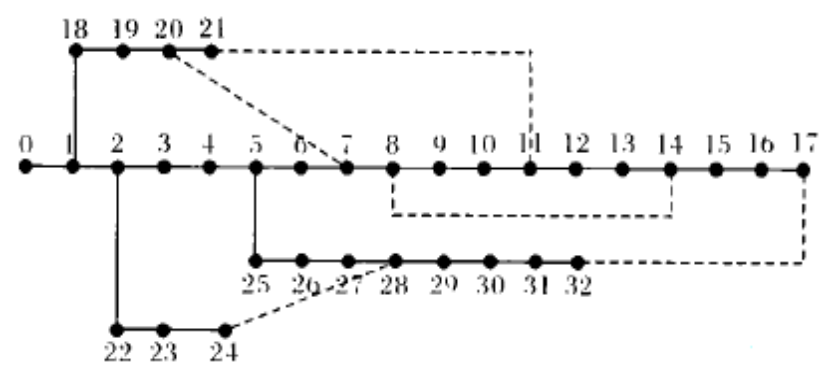

Figure 2. The topology of IEEE 33-bus power distribution system

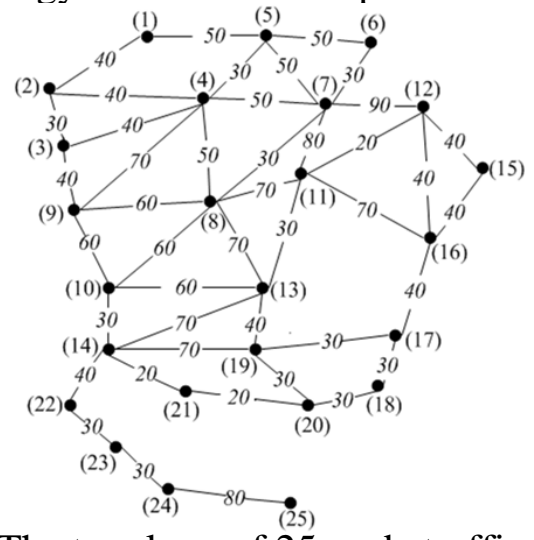

Figure 3. The topology of 25-node traffic system

\section{Results}

The population size of NSGA- II algorithm we used in this paper is set as 50 . The captured traffic flow (which we multiply with -1 to change maximizing to minimizing), initial population and bus voltage deviation of the initial population that randomly chose are shown in the 3-dimention figure 4(a). After 60 iterations, values of the above-mentioned 3 objectives of the final population 
are show in figure 4(b). It is obvious that after optimization, all the 3 objective values of the population become better, which shows the effectiveness of the proposed method.

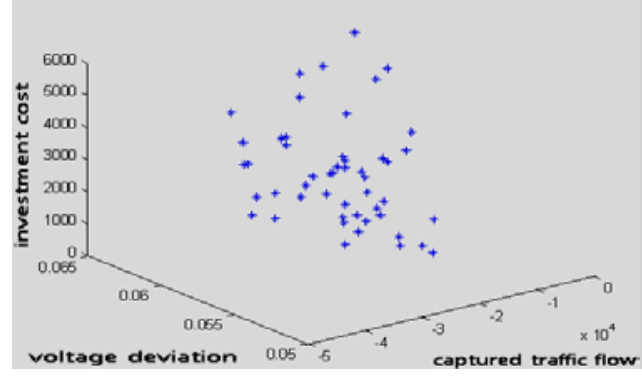

(a)

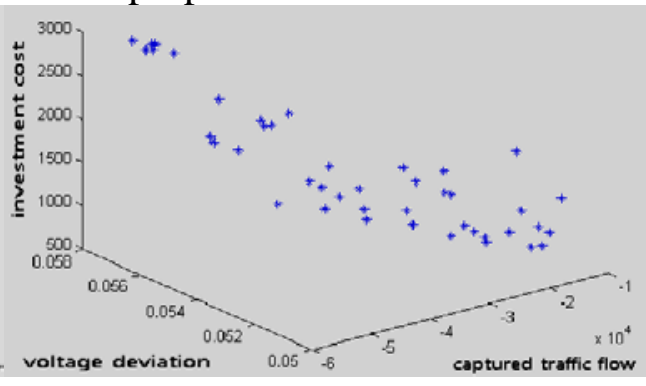

(b)

Figure 4. The objective values of the initial and optimized population

By using NSGA- II algorithm, we can have multiply pareto solutions simultaneously, which are widely spread in the feasible region. We choose the solutions solved once to illustrate the result. In this solution, the total traffic flow of the traffic network is $7.29 \times 10^{4}$ units. When 5 stations are built, the captured traffic flow is $5.57 \times 10^{4}$ units, which is $76.4 \%$ of the total flow. Under the circumstance that the charging stations capture most traffic flow, the positons of 5 stations are shown in figure 5.

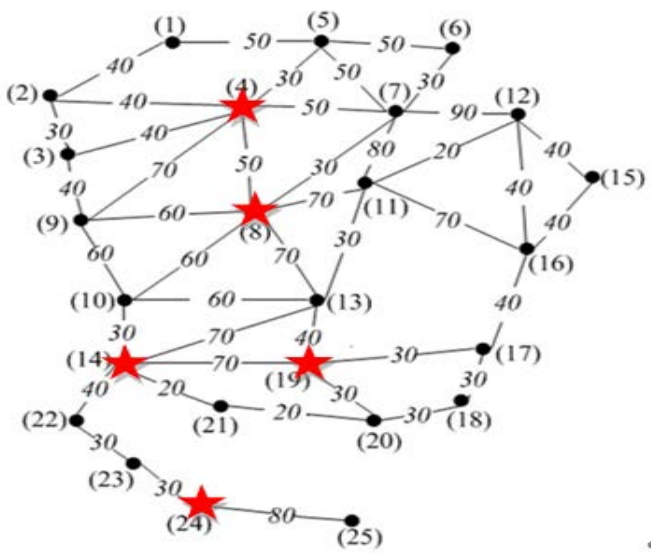

Figure 5. The positions of charging stations when they capture most traffic flow

We can find in figure 5, all the charging stations are built in the middle and west areas, the reason is there are more roads in these areas and accordingly, the traffic flow is more than east area. So when the total number of stations are not too many (in our test we only build 5 stations), they are mainly built in middle and west areas. Especially, although the roads from node 14 to node 25 are in the corner of the map, but in our test case, the weights of node 24 and 25 are very high in the simple gravity spatial interaction model we use, which means the traffic flow on these paths taking node 24 and 25 as destinations are quite much. In order to capture these traffic flow, it is easy to understand that we build charging stations at node 14 and 24 . Whilst node 14 is the only way vehicles can get to node 24 and 25, much traffic flow will pass here, based on the fact that the charging power of a station is proportional to the traffic flow it captured in our model, we can predicted the charging power of the station at node 14 will be very high. By assuming that the total charging power in the whole area is $800 \mathrm{kWh}$ and calculating the charging power of every stations, the results are as followed.

$$
\begin{aligned}
& P_{c, 4}=134 \mathrm{~kW} \\
& P_{c, 8}=190 \mathrm{~kW} \\
& P_{c, 14}=219 \mathrm{~kW} \\
& P_{c, 19}=98 \mathrm{~kW} \\
& P_{c, 24}=159 \mathrm{~kW}
\end{aligned}
$$

When we lay more importance on the investment cost, it is a little difficult to analyze the results because the investment cost contains construction cost, power expansion cost and power loss cost. Generally speaking, we need to consider the 3 aspects comprehensively. By solving the model, the cost is lowest when we build charging stations at node 1 , node 2 , node 6 , node 15 and node 19. 
When more attention is paid on bus voltage deviation, because the power distribution network is usually operated in radial topology, we can have the general idea that the voltage deviation is lower when the charging stations are built at the beginning nodes. We can observe from the results that the 5 charging stations are built at node 1 , node 2, node 19, node 20 and node 21 to lower the voltage deviation, and the deviation under this situation is 0.502 . The bus voltage of every power bus is shown in figure 6 when the voltage at swing bus 1 is set as $12.66 \mathrm{kV}$.

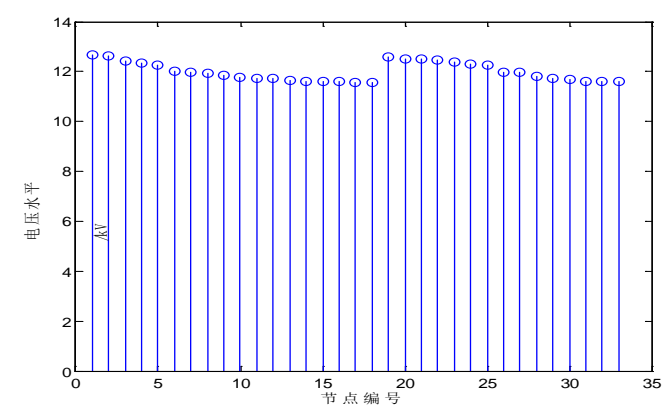

Figure 6. The bus voltages when the deviation is lowest

By analyzing the results as above, we can see the population is optimized in all three objective dimensions after we solve the model using NSGA- II algorithm. At the same time, we should also notice that because NSGA- II algorithm is an intelligent optimization method based on randomly searching, it is not certain that we can find the global optimal solutions every time. But it can help us find the most accurate solution by solving the problem several times and analyze the results from all the solutions we get.

\section{Conclusion}

By optimally planning the EV charging stations, we can better serve the EV owners and at the same time help the power distribution system keep stable. In this paper, the EV charging station placement model aiming at maximize the captured traffic flow, minimizing the investment cost and minimizing the bus voltage deviation is formulated. By solving the model using NSGA- II algorithm, we can get a set of pareto solutions, from which the decision maker can choose the most suitable one.

Based on this paper, there are several ways that we can improve. For example, we can further improve our model by considering more than shortest paths and second shortest paths. Also, we can modify the crossover and mutation operators in NSGA- II algorithm to shorten the solving time.

\section{References}

[1] XU Fan, Yu Guoqin, Gu Linfeng, et al. Tentative Analysis of Layout of Electrical Vehicle Charging Stations [J]. East China Electric Power, 2009 (10): 1678-1682.

[2] Lam A, Leung Y W, Chu X. Electric vehicle charging station placement[C]//Smart Grid Communications (SmartGridComm), 2013 IEEE International Conference on. IEEE, 2013: 510-515.

[3] Zakariazadeh A, Jadid S, Siano P. Multi-objective scheduling of electric vehicles in smart distribution system[J]. Energy Conversion and Management, 2014, 79: 43-53.

[4] WANG Hui, WANG Guibin, ZHAO Junhua, et al. Optimal Planning for Electric Vehicle Charging Stations Considering Traffic Network Flows [J]. Automation of Electric Power System, 2013, 37(13): 63-69.

[5] Hodgson M J. A Flow - Capturing Location - Allocation Model[J]. Geographical Analysis, 1990, 22(3): 270-279.

[6] Kuby M, Lim S. The flow-refueling location problem for alternative-fuel vehicles [J]. SocioEconomic Planning Sciences, 2005, 39(2): 125-145.

[7] HU Jiezhou. An Algorithm for Distribution Center Based on the Floyd-Shortest-Path [J]. Journal of Hunan Agricultural University (Natural Sciences), 2004, 30(4): 382-384. 
[8] CHAI Dengfeng, ZHANG dengrong. Algorithm and its application to N shortest paths problem [J]. Journal of Zhejiang University (Engineering Science). 2002, 36(5): 531-534.

[9] Kim J G, Kuby M. The deviation-flow refueling location model for optimizing a network of refueling stations[J]. international journal of hydrogen energy, 2012, 37(6): 5406-5420.

[10] Deb K, Pratap A, Agarwal S, et al. A fast and elitist multiobjective genetic algorithm: NSGA-II[J]. Evolutionary Computation, IEEE Transactions on, 2002, 6(2): 182-197 